\title{
ARTICLE Magnetic seizure therapy (MST) for major depressive disorder
}

Zafiris J. Daskalakis', Julia Dimitrova', Shawn M. McClintock², Yinming Sun', Daphne Voineskos', Tarek K. Rajji', David S. Goldbloom', Albert H. C. Wong ${ }^{1}$, Yuliya Knyahnytska $\mathbb{C}^{1}$, Benoit H. Mulsantt ${ }^{1}$, Jonathan Downar $\mathbb{D}^{3}$, Paul B. Fitzgerald ${ }^{4}$ and Daniel M. Blumberger ${ }^{1}$

Electroconvulsive therapy (ECT) is effective for major depressive disorder (MDD) but its effects on memory limit its widespread use. Magnetic seizure therapy (MST) is a potential alternative to ECT that may not adversely affect memory. In the current trial, consecutive patients with MDD consented to receive MST applied over the prefrontal cortex according to an open-label protocol. Depressive symptoms and cognition were assessed prior to, during and at the end of treatment. Patients were treated two to three times per week with high-frequency MST (i.e., $100 \mathrm{~Hz})(N=24)$, medium frequency MST (i.e., 60 or $50 \mathrm{~Hz})(N=26)$, or low-frequency MST (i.e., $25 \mathrm{~Hz}$ MST) $(N=36)$ using $100 \%$ stimulator output. One hundred and forty patients were screened; 86 patients with MDD received a minimum of eight treatments and were deemed to have an adequate course of MST; and 47 completed the trial per protocol, either achieving remission (i.e., 24-item Hamilton Rating Scale for Depression score $<10$ and a relative reduction of $>60 \%$ at two consecutive assessments; $n=17)$ or received a maximum of 24 sessions $(n=30)$. High-frequency $(100 \mathrm{~Hz}) \mathrm{MST}$ produced the highest remission rate (33.3\%). Performance on most cognitive measures remained stable, with the exception of significantly worsened recall consistency of autobiographical information and significantly improved brief visuospatial memory task performance. Under open conditions, MST led to clinically meaningful reduction in depressive symptoms in patients with MDD and produced minimal cognitive impairment. Future studies should compare MST and ECT under double-blind randomized condition.

Neuropsychopharmacology (2020) 45:276-282; https://doi.org/10.1038/s41386-019-0515-4

\section{INTRODUCTION}

Major depressive disorder (MDD) is a leading cause of global disease burden. It produces functional impairment and is associated with significant morbidity and mortality [1, 2]. About $33 \%$ of patients with MDD achieve remission of their depressive symptoms after a single trial of antidepressant medication. Even with multiple medication trials, $30-40 \%$ of patients do not improve significantly [3] and are categorized as suffering from treatment resistant depression (TRD).

Electroconvulsive therapy (ECT) is the most effective treatment for TRD with remission rates between 40 and 70\% [4]. However, $<1 \%$ of patients with TRD receive ECT [5]. Patients, families, and healthcare providers often do not consider ECT as a treatment option due to fear, stigma, and concerns regarding adverse cognitive effects associated with ECT [5]. Thus, there is a need for new treatment alternatives for patients with MDD. One such option is magnetic seizure therapy (MST).

In ECT, shunting of electrical current by the scalp and skull and conduction of electrical current through the cerebrospinal fluid results in a nonfocal and large total electrical charge delivered to the brain [6]. Such diffuse activation may be the mechanism through which neurocognitive adverse effects (e.g., memory impairment) occurs. By contrast, the magnetic fields used in MST are unimpeded and thus focal, requiring a lower total electrical charge to elicit an effective seizure [6]. This may account for the reports of absent or minimal effects of MST on neurocognitive function [7].
Early research suggested that MST is safe, well-tolerated, and has antidepressant effects $[8,9]$. Kayser et al. combined the results of a randomized clinical trial and an open-label trial of $100 \mathrm{~Hz}$ MST in a total of 26 patients with MDD, the response rate was $69 \%$ and the remission rate, $48 \%$ [10]. There were no significant adverse neurocognitive effects. Another study investigated the effects of MST in 13 patients in an open-label clinical trial of up to 18 treatment sessions with $100-\mathrm{Hz}$ MST [11]. Overall, depression severity decreased and $\sim 38 \%$ of the patients who completed the study met response criteria; the neurocognitive effects were benign. Lastly, Fitzgerald et al. [12] published on 37 patients who completed a course of at least nine ECT or MST treatments in a randomized double-blind protocol. They found that there was no clinically significant difference between MST and ECT and also reported no significant differences between the two treatments on cognitive performance.

In addition to their small samples, all these MST studies had limitations. They assessed $100 \mathrm{~Hz}$ delivered to the vertex. However, some evidence suggests that lower frequencies may be more effective at inducing seizures. For example, animal data show that the optimal frequency for seizure induction with MST is $\sim 22 \mathrm{~Hz}$ [13]. Targeting the vertex may also limit antidepressant efficacy as the pathophysiology of depression is closely associated with prefrontal neurocircuitry [14]. Finally, the total number of treatments was relatively short (e.g., up to 15). Thus, we conducted a study to evaluate the antidepressant and neurocognitive effects of MST applied at low, medium, and high frequencies over the

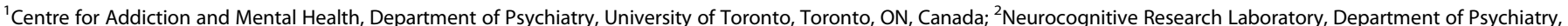

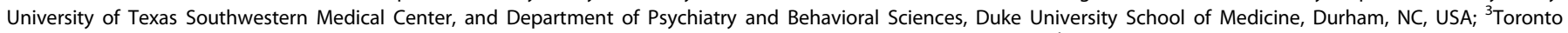

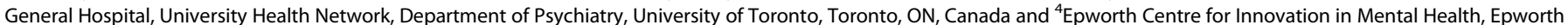
Healthcare and Monash Alfred Psychiatry Research Centre, The Alfred and Monash University Central Clinical School, Commercial Rd Melbourne, VIC, Australia Correspondence: Zafiris J. Daskalakis (Jeff.Daskalakis@camh.ca)

Received: 16 May 2019 Revised: 8 August 2019 Accepted: 21 August 2019

Published online: 5 September 2019 
prefrontal cortex for up to 24 treatments. We hypothesized that MST would produce significant rates of depressive symptom remission with negligible effects on cognition.

\section{METHODS}

Overall design

This study involved an open-label design with assessments of depression severity, subjective side-effects, and neurocognitive performance. We evaluated the efficacy of prefrontal MST at the following frequencies in three cohorts: (i) high-frequency MST (i.e., $100 \mathrm{~Hz}$ ); (ii) moderate frequency MST initially applied at $50 \mathrm{~Hz}$, then at $60 \mathrm{~Hz}$ - the highest frequency at which MST can be administered at $100 \%$ intensity continuously (without a roll-off effect on stimulation intensity); and (iii) low-frequency MST (i.e., $25 \mathrm{~Hz}$ ). The moderate frequency $60 \mathrm{~Hz}$ cohort was treated last in the sequence above.

Patients

Patients with MDD with or without psychotic features were recruited at the Center for Addiction and Mental Health (CAMH; Toronto, Ontario). The protocol was approved by the CAMH Research Ethics Board in accordance with the Declaration of Helsinki. All patients provided written informed consent. Patients were included if they: (1) had a DSM-IV-TR major depressive episode with or without psychotic features in the context of a diagnosis of MDD, (2) were referred for a course of ECT, (3) were 18-85-year-old, (4) had a 24-item Hamilton Rating Scale for Depression (HRSD-24) total score of $>21$, and (5) were on a medically-acceptable form of birth control, if a woman of child-bearing potential. Patients were excluded if they: (1) had unstable medical and/or neurological condition or were currently pregnant or lactating, (2) were not considered sufficiently physically stable to undergo general anesthesia, (3) had a cardiac pacemaker, cochlear implant, implanted electronic device, or non-electric metallic implant, (4) were taking a benzodiazepine at a dose greater than lorazepam $2 \mathrm{mg}$ or equivalent, (5) were taking any anticonvulsant medications, (6) had active substance misuse within the past 3 months, (7) had a diagnosis of delirium, dementia or a cognitive disorder secondary to a general medical condition, (8) had a lifetime diagnosis of an Eating Disorder, (9) had other significant neuropsychiatric comorbidity, (10) had a history of any suicide attempt in the past 6 months, and (11) met diagnostic criteria for antisocial or borderline personality disorder on the Structured Clinical Interview for DSM-IV-TR Axis II Personality Disorders (SCID-II). Eligible patients were treated at the Temerty Centre for Therapeutic Brain Intervention at $\mathrm{CAMH}$. If receiving a course of pharmacotherapy for their current depressive episode at the time of enrollment, patients were allowed to continue and instructed not make any changes during the duration of their enrollment. Information regarding concomitant medications was collected at baseline and after every three treatments to monitor for any changes.

Clinical measures

Demographic information, medical history, and concomitant medications were recorded at the screening visit. Baseline medical comorbidity was assessed with the Cumulative Illness Rating Scale [15]. Neuropsychiatric diagnosis was confirmed using the Structured Clinical Interview for the DSM-IV-TR (SCID-I), and depression severity was quantified with the HRSD-24. The Antidepressant Treatment History Form (ATHF) was used to document treatment resistance level $[16,17]$.

Depressive symptoms were assessed at baseline, after every three treatments, and at the end of the treatment course. Response was defined as $\geq 50 \%$ reduction in the HRSD- 24 total score from baseline to end of treatment. Remission was defined as a post HRSD- 24 total score $\leq 10$ and $\geq 60 \%$ relative decrease in total score from baseline on two consecutive ratings.

Neurocognitive assessment

Patients completed a comprehensive neurocognitive battery of measures that assessed multiple neurocognitive domains including global cognitive function, premorbid intellectual ability, processing speed, attention, learning and memory, working memory, autobiographical memory, and executive function. Specific instruments included the Montreal Cognitive Assessment Test (MoCA) [18], Wechsler Test of Adult Reading [19], Controlled Oral Word Association Test (COWAT) [20], and the MATRICS Consensus Cognitive Battery (MCCB) which includes the following tests: Category Fluency Test, Trail Making Test, Symbol Coding Test, Hopkins Verbal Learning Test-Revised (HVLT-R), Brief Visuospatial Memory Test-Revised (BVMT-R), Spatial Span Test, Letter Number Sequencing Span, Mazes Test [21]. The Stroop Neuropsychological Screening Test [22], and the Autobiographical Memory Interview Short Form (AMI-SF) [23] were also used. Alternate forms were used for the following assessments after the initial baseline data collection: MoCA (versions 7.1, 7.2, 7.3), HVLT-R (Forms 1, 3, 5), and BVMT-R (Forms 1, 2, 3).

Patients completed neurocognitive assessments at baseline and the end of the treatment course. In addition, time to reorientation was assessed after each MST treatment and followed published recommendations [24].

MST treatment procedure

MST was delivered over the frontal cortex using the MagPro MST device with a bilateral dual circular coil, the Twin Coil (Twin CoilXS). At the first MST session, the seizure threshold was titrated using a predefined dosing schedule. During titration, a maximum of three stimulations were administered in the same session provided that the coil temperature allowed for a third stimulation. If an adequate seizure was not produced by the third stimulation, titration continued at the next treatment session until threshold was reached. Titration schedules were determined based on the frequency of stimulation and the MST device stimulation capacity. If stimulation was administered at 50,60 , or $100 \mathrm{~Hz}$, titration would begin with $2 \mathrm{~s}$ of stimulation and increase in increments of $2 \mathrm{~s}$, until a maximum stimulus of 1000 pulses was reached (Table 1).

\begin{tabular}{|c|c|c|c|c|}
\hline & \multicolumn{4}{|c|}{ Frequency } \\
\hline & $100 \mathrm{~Hz}$ & $60 \mathrm{~Hz}$ & $50 \mathrm{~Hz}$ & $25 \mathrm{~Hz}$ \\
\hline Duration & \multicolumn{4}{|c|}{ Number of pulses } \\
\hline 2 & 200 & 120 & 100 & \\
\hline 4 & 400 & 240 & 200 & \multirow[t]{2}{*}{100} \\
\hline 6 & 600 & 360 & 300 & \\
\hline 8 & 800 & 480 & 400 & \multirow[t]{2}{*}{200} \\
\hline 10 & \multirow[t]{6}{*}{1000} & 600 & 500 & \\
\hline 12 & & 720 & 600 & \multirow[t]{2}{*}{300} \\
\hline 14 & & 840 & 700 & \\
\hline 16 & & 960 & 800 & \multirow[t]{2}{*}{400} \\
\hline 18 & & & 900 & \\
\hline 20 & & & 1000 & 500 \\
\hline \multicolumn{5}{|c|}{$\begin{array}{l}\text { MST was administered at maximum stimulator output ( } 100 \% \text { intensity), } \\
\text { although there is a roll-off effect at frequencies higher than } 60 \mathrm{~Hz} \text {. The } \\
\text { maximum numbers of pulses administered in a single stimulation was } \\
1000 \text { pulses based on device operating specifications. For } 25 \mathrm{~Hz} \text {, the } \\
\text { maximum was } 500 \text { pulses or } 20 \mathrm{~s} \text {, as this is the maximum duration at which } \\
\text { the device could provide continuous stimulation }\end{array}$} \\
\hline
\end{tabular}


The dose was adjusted based on treatment response. Similarly, at $25 \mathrm{~Hz}$ stimulation, titration would begin with $4 \mathrm{~s}$ of stimulation and increase in increments of $4 \mathrm{~s}$, until the maximum dose of 500 pulses was reached. After the titration session, stimulation was administered at a dose three times that of the initial seizureproducing threshold. If the patient had no seizure, another stimulation would be administered at the next dose on the titration schedule. If maximum dose was applied (i.e., 1000 or 500 pulses), a second stimulation using the same parameters was administered during the same session. Patients were treated two to three times per week until they achieved depressive symptom remission or completed a maximum of 24 MST sessions.

\section{Anesthesia}

Anesthesiologists experienced in ECT administered general anesthesia, with all patients started on methohexital $(0.375-0.75 \mathrm{mg} / \mathrm{kg} \mathrm{IV})$ and succinylcholine $(0.5-1.0 \mathrm{mg} / \mathrm{kg}$ IV) and mask ventilation with $100 \%$ oxygen. For patients with inadequate seizures as determined by the trained study psychiatrist, the dose of methohexital was decreased and remifentanil $(1.0-1.5 \mathrm{mcg} / \mathrm{kg})$ was added as a second anesthetic agent as used in convulsive therapy practice. Two patients were switched to ketamine as the sole anaesthestic agent due to inadequate seizures. Monitoring of blood pressure, oxygen saturation, heart rate, and EKG occurred throughout each session following standard clinical guidelines.

\section{Data Analysis}

All statistical analyses were conducted using the SPSS 24 statistical software (IBM Inc.). The primary analysis included patients who completed a minimally adequate trial of MST, defined as at least 8 sessions (i.e., adequate trial completers) [25]. In addition, a perprotocol analysis included patients who completed the protocol (i.e., protocol completers) by either completing the maximum of 24 sessions or achieving the defined criteria for remission. Baseline differences in demographic and clinical variables were compared between treatment groups. Continuous variables, i.e., ATHF scores, seizure duration, and time to reorientation, were analyzed with one-way analysis of variance (ANOVA). Categorical variables (i.e., proportions of responders and remitters) were analyzed with $x^{2}$ analyses, i.e., proportions of responders and remitters. All analyses were two-tailed with a significance level set at $a=0.05$. Neurocognitive outcomes were analyzed using paired samples $t$ tests for baseline and post-treatment data. In order to minimize false positive findings due to overinflation of the alpha values due to the large number of comparisons, neurocognitive findings were considered to be significant at $a=0.005$.

\section{RESULTS}

Patient flow, sample characteristics, and follow-up

Of the 140 patients consented and screened for eligibility, 24 did not meet inclusion criteria, 6 declined participation, 2 did not receive treatment due to a device malfunction, 15 withdrew from the study, and 7 were discontinued prior to completing an adequate MST course. Thus, the primary analysis included 86 patients with MDD who completed a minimally adequate MST course (i.e., at least 8 sessions). There were 47 patients who were protocol completers (see CONSORT Diagram). The patients' baseline demographic and clinical characteristics are summarized in Table 2. A one-way ANOVA revealed no significant differences between the three frequency groups on age, baseline HRSD score, years of education, number of previous depressive episodes, duration of the current episode, and age at onset of depressive illness. Chi-square analyses revealed a significant difference between the moderate and high-frequency group on number of individuals endorsing a recurrent episode of depression, with a significantly higher proportion in the high-frequency group. No other significant differences were found.

\section{Antidepressant treatment history}

Adequate trial completers. Among the patients who completed an adequate trial, a mean $( \pm S D)$ of $5.57( \pm 2.95)$ medication trials was recorded for the current episode on the ATHF. The mean number of medication trials for the current episode was 5.86 $( \pm 2.98)$ for the low-frequency MST group, $6.60( \pm 2.93)$ for the moderate frequency MST group, and $3.96( \pm 2.29)$ in the highfrequency MST group. There was a significant difference between the stimulation frequency groups for the mean number of medication trials recorded for the current episode $(F(2,76)=$ $7.19, p=0.001)$. There was also a significant difference between groups for number of adequate antidepressant trials in the current

Table 2. Demographic and clinical characteristics

\begin{tabular}{|c|c|c|c|c|c|c|}
\hline DSM IV-TR Diagnosis-MDD (n) & 86 & 36 & 26 & 24 & & \\
\hline Sex (\% females) & 57.4 & 53.5 & 59.5 & 61.5 & & $\mathrm{~ns}^{\mathrm{a}}$ \\
\hline Education (years) & $15.31(2.95)$ & $15.21(2.64)$ & $15.39(2.96)$ & $15.58(3.38)$ & 0.57 & \\
\hline Baseline HRSD & & & & & 0.42 & \\
\hline Length of current MDE (weeks) & $178.54(182.07)$ & $170.89(18.23)$ & $225.35(195.59)$ & $125.15(152.69)$ & 0.22 & \\
\hline Recurrent MDE (\% yes) & 77.8 & 77.8 & 70.3 & 88.5 & & $3.93^{\mathrm{b}}$ \\
\hline ATHF cumulative strength rating (current MDE) & $13.46(8.85)$ & $14.89(9.50)$ & $15.65(8.76)$ & $8.16(5.07)$ & $5.20^{*}$ & \\
\hline Total medication trials (current MDE) & $5.57(2.95)$ & $5.86(2.98)$ & $6.60(2.93)$ & $3.96(2.29)$ & $7.20^{* *}$ & \\
\hline
\end{tabular}


episode, whereby patients in the low-frequency group $(p=0.03)$ and moderate frequency group $(p=0.009)$ had significantly higher adequate antidepressant trials than patients in the highfrequency group.

Protocol completers. Among the patients who completed the trial as per protocol, a mean $( \pm S D)$ of $5.36( \pm 2.51)$ medication trials was recorded for the current episode on the ATHF. The mean $( \pm S D)$ number of medication trials for the current episode was 5.32 $( \pm 2.11)$ for the low-frequency MST group, $6.80( \pm 2.94)$ for the moderate frequency MST group and $4.47( \pm 2.39)$ in the highfrequency MST group. There was no significant difference between the stimulation frequency groups for the mean number of medication trials recorded for the current episode $(F(2,41)=$ $2.83, p=0.07)$. There was also no significant difference between groups for number of adequate antidepressant trials in the current episode $(F(2,29)=2.98, p=0.07)$.

EEG seizure duration

EEG seizure duration was calculated in patients who completed an adequate trial of MST as the mean duration of the second treatment and the last treatment. The mean $( \pm S D)$ seizure duration across all three groups was $46.74( \pm 22.51 \mathrm{~s})$; for the high-frequency group, it was $41.66( \pm 22.57) \mathrm{s}$; for the moderate frequency group, $51.19( \pm 22.34) \mathrm{s}$; and for the low-frequency group, $46.64( \pm 22.56 \mathrm{~s})$ : these durations did not differ significantly between the three groups $(F(2,81)=1.07, p=0.35)$. Readers are referred elsewhere for a detailed analysis of other seizure characteristics (e.g., post-ictal suppression, global seizure strength, polyspike amplitude, etc.) in this sample [26].

\section{Antidepressant effects}

Adequate trial completers. High-frequency MST produced the largest response rate ( 10 of $24 ; 41.7 \%$ ), followed by low (12 of 36; $33.3 \%$ ), and moderate (7 of 26; $26.9 \%$ ) frequency MST. These rates did not differ significantly. Also, high-frequency MST produced the highest rates of remission ( 8 of $24 ; 33.3 \%$ ) followed by moderate ( 5 of $26 ; 19.2 \%$ ) and low (4 of 36; $11.1 \%$ ) frequency MST; only the remission rate with high-frequency MST was significantly higher that the remission rate with low-frequency MST $\left(X^{2}(1)=4.44, p=\right.$ 0.04).

Protocol completers. High-frequency MST produced the highest response rate ( 9 of $15 ; 60 \%)$, followed by low (11 of $19 ; 57.9 \%$ ), and moderate (7 of $13 ; 53.8 \%$ ) frequency MST; these rates did not differ significantly. Also, high-frequency MST produced the highest remission rate $(8$ of $15 ; 53.3 \%)$, followed by moderate ( 5 of $13 ; 38.5 \%$ ), and low (4 of $19 ; 21.1 \%$ ) frequency MST; the remission rates with high-frequency MST were significantly higher that with low-frequency MST $\left(X^{2}(1)=3.825, p=0.05\right)$. See Figs. 1 and 2.

Time to remission

The mean $( \pm S D)$ number of treatments associated with remission was $15.71 \pm 5.76$ across all three frequency groups. The mean number of treatments associated with remission in the highfrequency group was $14.50+5.17,17.40+3.91$ for the moderate frequency group was, and $15.50 \pm 7.92$ for the low-frequency group. There were no significant differences between the three groups $(F(2,14)=0.32, p=0.73)$.

Time to reorientation

Reorientation time was calculated in adequate trial completers as the mean time between the second treatment and the last treatment. The overall mean $( \pm S D)$ reorientation time across all three groups was $10.52 \pm 7.52 \mathrm{~min}$. The mean $( \pm S D)$ reorientation time was $3.99 \pm 4.07 \mathrm{~min}$ for the high-frequency group, $13.44 \pm 7.48$ minutes for the moderate frequency group, and

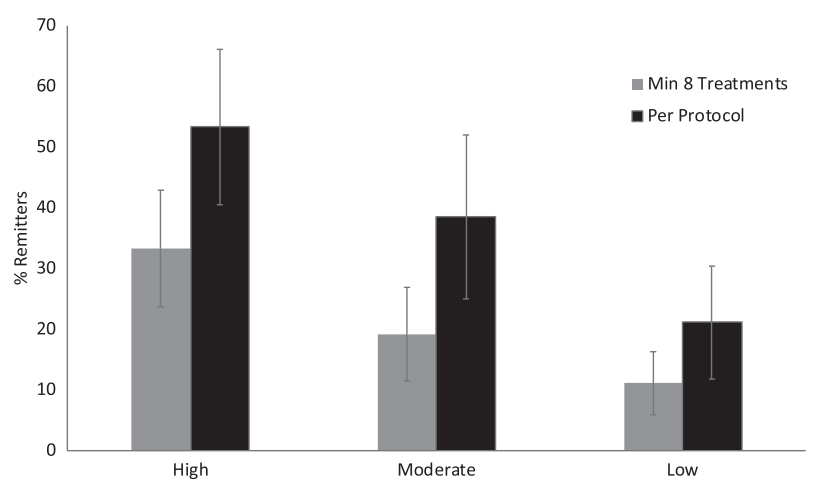

Fig. 1 Percentage of remitters based on treatment frequency received and number of treatments completed. The error bars attached to each column represent standard error

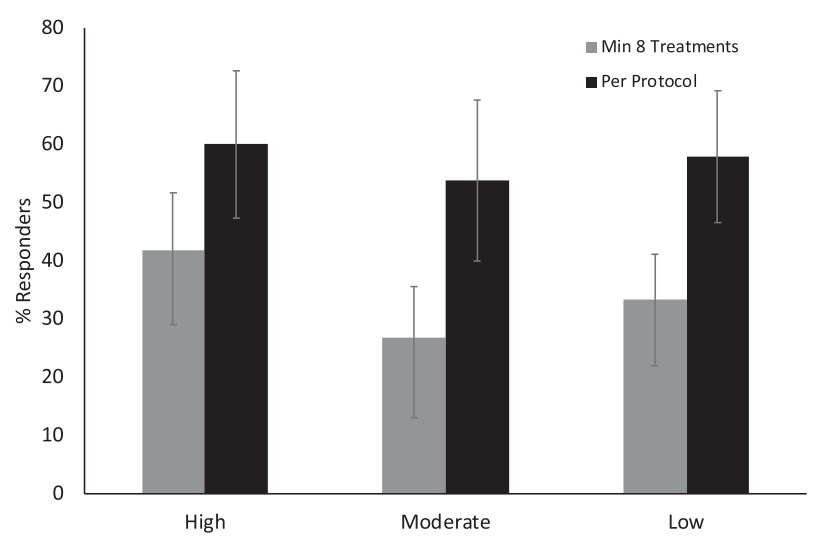

Fig. 2 Percentage of responders based on treatment frequency received and number of treatments completed. The error bars attached to each column represent standard error

$12.40 \pm 6.91 \mathrm{~min}$ for the low-frequency group. These reorientation times differed significantly between the three groups $(F(2,81)=$ $15.25, p<0.001)$; reorientation times were shorter with highfrequency stimulation than with moderate frequency $(p<0.001)$ and low frequency $(p<0.001)$ otherwise there were no significant differences in reorientation times.

\section{Neurocognitive function}

Adequate trial completers. Across all patients, there was a significant decrease from baseline to end on the AMI-SF scores $(t(71)=17.56, p<0.001)$. Consistency loss using AMI-SF scores was also examined based on the percentage of autobiographical recall inconsistency from the baseline score. When comparing post treatment to baseline in our sample, we found an average of $19 \%$ consistency loss. The patients receiving low-frequency MST had a consistency loss of $16 \%$, while patients in the moderate frequency MST and high-frequency MST groups had a consistency loss of 28 and $17 \%$, respectively. A one-way ANOVA revealed a significant difference between treatment frequency on consistency loss $(F(2$, $69)=9.42, \quad p<0.001)$, with the moderate frequency group presenting with significantly more consistency loss as compared to the low $(p<0.001)$ and high $(p=0.003)$ frequency groups. There was a significant positive association $(r=0.42)$ between consistency loss on the AMI and mean time to reorientation in the overall sample. Within groups, this association was significant for the low-frequency MST group $(r=0.44)$, but not the moderate and high-frequency MST groups. There also was a significant increase in the BVMT-R total recall $(t(70)==3.92, p<0.001)$, and delayed recall scores $(t(70)=-4.32, p<0.00$; Table 3$)$. 
Table 3. Means and SD across groups for neurocognitive measures among patients who have completed an adequate trial of MST ( $\geq$ eight treatments) or per protocol

\begin{tabular}{|c|c|c|c|c|c|c|}
\hline \multirow[b]{2}{*}{ Measure } & \multicolumn{3}{|l|}{ Adequate trial } & \multicolumn{3}{|l|}{ Per protocol } \\
\hline & $\mathrm{M}(\mathrm{SD})$ & $M(S D)$ & $t$ & $\mathrm{M}(\mathrm{SD})$ & $M(S D)$ & $t$ \\
\hline MoCA & $25.73(3.63)$ & $25.96(3.5)$ & -0.635 & $25.88(3.78)$ & $26.02(3.06)$ & -0.504 \\
\hline AMI-SF & $51.42(6.89)$ & $41.58(8.61)$ & $17.56^{*}$ & $51.16(7.4)$ & $40.98(9.1)$ & $12.875^{*}$ \\
\hline Trails A & $40(33.3)$ & $34.05(14.27)$ & 1.183 & $40.4(31.52)$ & $36.38(15.94)$ & 0.076 \\
\hline HVLT-R total & $25.26(6.29)$ & $24.67(5.2)$ & 2.059 & $25.53(6.64)$ & $24.32(5.5)$ & 2.399 \\
\hline HVLT-R recall & $8.78(2.93)$ & $8.53(3)$ & 1.661 & $8.93(3)$ & $8.34(3.33)$ & 2.233 \\
\hline HVLT-R recognition index & $10.53(1.93)$ & $10.73(1.66)$ & -0.571 & $10.8(1.53)$ & $10.57(1.82)$ & 1.277 \\
\hline Spatial span forward & $8.47(2)$ & $8.58(1.99)$ & 0.177 & $8.53(1.85)$ & $8.41(1.92)$ & 0.561 \\
\hline Spatial span back & $7.78(2.06)$ & $7.93(1.99)$ & 0.575 & $7.76(1.92)$ & $7.41(1.93)$ & 1.677 \\
\hline BVMT-R total & $21.8(8.78)$ & $25.39(7.41)$ & $-3.923^{*}$ & $22.6(8.29)$ & $25.1(7.73)$ & -2.709 \\
\hline BVMT-R learning & $3.94(2.16)$ & $3.96(2.23)$ & 0.307 & $3.84(2.26)$ & $4(2.18)$ & -0.282 \\
\hline BVMT-R recall & $8.4(3.05)$ & $9.85(2.49)$ & $-4.318^{*}$ & $8.65(2.84)$ & $9.6(2.59)$ & -2.506 \\
\hline BVMT-R recognition index & $5.58(0.81)$ & $5.81(0.49)$ & -2.114 & $5.58(0.73)$ & $5.83(0.44)$ & -1.939 \\
\hline COWAT total & $39.06(14.55)$ & $37.47(14.36)$ & 1.458 & $39.58(16.2)$ & $37.1(15.49)$ & 2.315 \\
\hline Categories total & $54.63(19.4)$ & $51.14(20.66)$ & 2.69 & $55.35(20.89)$ & $53.07(20.15)$ & 2.4 \\
\hline Stroop solor-word score & $95.19(22.25)$ & $95.8(22.02)$ & 0.493 & $95.19(22.02)$ & $94.63(22.92)$ & 0.299 \\
\hline Stroop color-word time (sec) & $113.62(16.23)$ & $111.09(17.92)$ & 2.282 & $115.4(17.85)$ & $115.8(17.3)$ & 0.407 \\
\hline
\end{tabular}

Protocol completers. Across all patients, there was a significant decrease from baseline to end on the AMI-SF; it was consistently found in all three MST frequency conditions, $t(43)=12.88$, $p<0.001$; Table 3).

\section{Safety}

Among the 140 patients who signed consent and were screened for eligibility, there were 17 serious adverse events (SAEs) that affected $16(11.4 \%)$ patients. SAEs that were deemed related or possibly related to MST included the emergence of mania $(n=1)$, hospitalization related to a fall and dislocated shoulder $(n=1)$, and a superficial burn on the head due to a coil malfunction $(n=1)$.

\section{DISCUSSION}

MST was associated with a significant reduction in depressive symptoms in patients with MDD, both in adequate trial and protocol completers. The effect of MST was more pronounced in the high-frequency stimulation group than in the low and moderate frequency stimulation groups. There were no changes in cognition except for a reduction in autobiographical memory (AMI-SF scores) and an improvement in visuospatial memory (BVMT-R scores) and delayed recall scores. Also, postictal orientation times were significantly shorter in the highfrequency group.

Our study was primarily limited by a lack of an active comparator group. However, several important factors mitigate this limitation. First, the remission and response rates observed in the $100 \mathrm{~Hz}$ MST group are higher than that observed in MDD patients with other forms of electromagnetic brain stimulation. For example, in a recent meta-analysis, Berlim et al. [27] reported an rTMS remission rate of $18.6 \%$ with active treatment and $5 \%$ with sham stimulation in over 15 randomized controlled trials (RCTs) and 450 participants. By contrast, in patients treated with highfrequency MST, we observed a remission rate of $32 \%$ in the adequate trial completers and $53.3 \%$ in the protocol completers. Right unilateral ultrabrief ECT, the form of ECT associated with the fewest cognitive effects, has a remission rate of $35.29 \%$ [25] - comparable to our remission rates, but lower than the $61.7 \%$ remission rates of the multicenter Prolonging Remission in Depressed Elderly (PRIDE) study [28]. It is important to note, however, that the PRIDE study involved only patients with geriatric depression. Also, in the PRIDE trial, a minimum of 12 treatments was considered to be an adequate course.

As reported earlier, previous MST treatment trials have yielded variable results (e.g., Kayser et al. [10] reported a $46 \%$ remission rate and Fitzgerald et al. [11] reported a 15\% remission rate). There are key differences between these trials and ours. These differences include site of stimulation (i.e., frontal versus vertex) and the number of treatments administered (up to 18 in Kayser et al. [10], up to 15 in Fitzgerald et al. [12], and up to 24 in our trial). We cannot conclude whether there are any benefits to frontal stimulation compared with vertex stimulation. By contrast, it appears that longer courses of treatment (as in both the Kayser et al. studies and our study) are associated with higher remission rates. 
Another limitation of our study is the consecutive (nonrandom) allocation of patients to the different frequency groups. The low and moderate frequency groups had a higher total number of antidepressant medications trials for the current episode than the high-frequency group. However, even though these differences were not significant in the protocol completers, differences in clinical outcomes between the high and low-frequency groups remained.

While our overall findings suggest that MST does not affect cognitive performance, three findings survived correction for multiple comparisons. First, there were significant decreases in AMI-SF scores in all three frequency groups. The AMI-SF is the short form version of the Autobiographical Memory Interview [23]. This measure has recently been criticized due to the lack of normative data from healthy control samples or appropriately matched depressed non-ECT samples, and the absence of reliability and validity studies [29]. However, proponents of the AMI-SF have reported on its sensitivity in differentiating the effects of different forms of ECT (e.g., bitemporal versus right unilateral electrode configuration) on retrograde autobiographical amnesia (RAA). Kessler et al. [30] compared the effects of right unilateral ECT to pharmacotherapy on neurocognitive function. Pharmacotherapy alone produced a decay of autobiographical memory consistency loss of $19 \%$ on the AMI, significantly better than the ECT group of $27 \%$ consistency loss, and equivalent to the overall AMI consistency loss of $19 \%$ in our trial. Given that there is a natural and welldocumented loss that characterizes normal autobiographical memory function, and that the AMI-SF is a psychometrically unsound measure, it is difficult to characterize any decreases on the AMI-SF as conclusive evidence of retrograde amnesia induced by MST. Importantly, from baseline to post-treatment, performance across all other cognitive measures remained stable without clinically meaningful changes, except there was improvement in visuospatial memory. Collectively, the unadjusted and multiple comparison adjusted findings suggests that MST is cognitively safe, which is consistent with prior research [31].

In conclusion, our results suggest that MST can be an effective treatment for some patients with MDD. On average, patients showed decreased recall consistency of autobiographical information that was likely related to an effect of time rather than treatment, whereas all other cognitive functions remained stable with improvement in visuospatial memory. MST also produced some improvement in visuospatial memory and delayed recall. MST could have a significant impact on the treatment of MDD if future studies demonstrate that its efficacy is comparable to the efficacy of ECT, while maintaining cognitive safety. To that end, we are currently conducting a randomized, non-inferiority, doubleblind trial evaluating the efficacy, tolerability, and cognitive adverse effects of MST to ECT (ClinicalTrials.gov Identifier: NCT03191058). If the efficacy of MST and ECT are comparable but MST has a more favorable cognitive effect profile, then MST may be preferred to ECT and more patients may agree to try this treatment.

\section{FUNDING AND DISCLOSURE}

The authors thank the clinical research staff and the patient participants of the study. In the last 5 years, ZJD has received research and equipment in-kind support for an investigator-initiated study through Brainsway Inc and Magventure Inc. His work was supported by the Ontario Mental Health Foundation, the Canadian Institutes of Health Research (CIHR), the National Institutes of Mental Health, and the Temerty Family and Grant Family and through the Centre for Addiction and Mental Health (CAMH) Foundation and the Campbell Institute. During the past five years, BHM has received: research funding from Brain Canada, the CAMH Foundation, the CIHR, and the US National Institutes of Health (NIH); research support from Bristol-Myers Squibb (medications for a $\mathrm{NIH}$-funded clinical trial), Eli-Lilly (medications for a $\mathrm{NIH}$-funded clinical trial), Pfizer (medications for a NIH-funded clinical trial), Capital Solution Design LLC (software used in a study funded by CAMH Foundation), and HAPPYneuron (software used in a study funded by Brain Canada). He directly own stocks of General Electric $(<\$ 5,000)$. DMB has received research support from $\mathrm{ClHR}$, $\mathrm{NIH}$, Brain Canada and the Temerty Family through the CAMH Foundation and the Campbell Research Institute. He receives research support and in-kind equipment support for an investigator-initiated study from Brainsway Ltd. and he is the site principal investigator for three sponsor-initiated studies for Brainsway Ltd. He also receives in-kind equipment support from Magventure for an investigator-initiated study. He received medication supplies for an investigator-initiated trial from Indivior. SMM has received research support from the NIH. He has received teaching honorarium from TMS Health Solutions. He is a consultant to Pearson. DV has received research training fellowship funding from the Ontario Mental Health Foundation, the APA Eli Lilly research fellowship and a CAMH Postdoctoral Fellowship, and support from the Innovation Fund of the Alternative Funding Plan for the Academic Health Sciences Centres of Ontario. JD reports research grants from $\mathrm{CIHR}$, the National Institute of Mental Health, Brain Canada, the Canadian Biomarker Integration Network in Depression, the Ontario Brain Institute, the Weston Foundation, the Klarman Family Foundation, the Arrell Family Foundation, and the Buchan Family Foundation, travel stipends from Lundbeck and ANT Neuro, in-kind equipment support for investigator-initiated trials from MagVenture, and is an advisor for BrainCheck, TMS Neuro Solutions, and Restorative Brain Clinics. TKR has received research support from Brain Canada, Brain and Behavior Research Foundation, BrightFocus Foundation, Canada Foundation for Innovation, Canada Research Chair, CIHR, Centre for Aging and Brain Health Innovation, NIH, Ontario Ministry of Health and Long-Term Care, Ontario Ministry of Research and Innovation, and the Weston Brain Institute. PBF is supported by a Practitioner Fellowship grant from National Health and Medical Research Council (1078567). In the last 3 years PBF has received equipment for research from Magventure A/S, Medtronic Ltd, Neurosoft and Brainsway Ltd. He has served on a scientific advisory board for Bionomics Ltd and LivaNova and is a founder and director of TMS Australia. YK has nothing to disclose. DG has nothing to disclose. ACHW has nothing to disclose. JD (Julia Dimitrova) has nothing to disclose. YS has nothing to disclose.

\section{ADDITIONAL INFORMATION}

Supplementary Information accompanies this paper at (https://doi.org/10.1038/ s41386-019-0515-4).

Publisher's note Springer Nature remains neutral with regard to jurisdictional claims in published maps and institutional affiliations.

\section{REFERENCES}

1. Patten SB, Kennedy SH, Lam RW, O'Donovan C, Filteau MJ, Parikh SV, et al. Canadian Network for Mood and Anxiety Treatments (CANMAT) clinical guidelines for the management of major depressive disorder in adults. I. Classification, burden and principles of management. J Affect Disord. 2009;117(Suppl 1):S5-14.

2. Lepine JP, Briley M. The increasing burden of depression. Neuropsychiatr Dis Treat. 2011;7:3-7.

3. Rush AJ, Trivedi MH, Wisniewski SR, Nierenberg AA, Stewart JW, Warden D, et al. Acute and longer-term outcomes in depressed outpatients requiring one or several treatment steps: a STAR*D report. Am J Psychiatry. 2006;163:1905-1917.

4. Group UER. Efficacy and safety of electroconvulsive therapy in depressive disorders: a systematic review and meta-analysis. Lancet. 2003;361:799-808.

5. Wilkinson ST, Agbese E, Leslie DL, Rosenheck RA. Identifying recipients of electroconvulsive therapy: data from privately insured Americans. Psychiatr Serv. 2018.

6. Deng ZD, Lisanby SH, Peterchev AV. Electric field strength and focality in electroconvulsive therapy and magnetic seizure therapy: a finite element simulation study. J Neural Eng. 2011;8:016007.

7. McClintock SM, Tirmizi O, Chansard M, Husain MM. A systematic review of the neurocognitive effects of magnetic seizure therapy. Int Rev Psychiatry. 2011;23:413-423.

8. Kosel M, Frick C, Lisanby SH, Fisch HU, Schlaepfer TE. Magnetic seizure therapy improves mood in refractory major depression. Neuropsychopharmacology. 2003:28:2045-2048.

9. Lisanby SH, Schlaepfer TE, Fisch HU, Sackeim HA. Magnetic seizure therapy of major depression. Arch Gen Psychiatry. 2001;58:303-305.

10. Kayser S, Bewernick BH, Matusch A, Hurlemann R, Soehle M, Schlaepfer TE. Magnetic seizure therapy in treatment-resistant depression: clinical, neuropsychological and metabolic effects. Psychol Med. 2015;45:1073-1092.

11. Fitzgerald $\mathrm{PB}$, Hoy KE, Herring SE, Clinton AM, Downey G, Daskalakis ZJ. Pilot study of the clinical and cognitive effects of high-frequency magnetic seizure therapy in major depressive disorder. Depress Anxiety. 2013;30:129-136.

12. Fitzgerald PB, Hoy KE, Elliot D, McQueen S, Wambeek LE, Chen L, et al. A pilot study of the comparative efficacy of $100 \mathrm{~Hz}$ magnetic seizure therapy and 
electroconvulsive therapy in persistent depression. Depress Anxiety. 2018:35:393-401.

13. Peterchev AV, Rosa MA, Deng ZD, Prudic J, Lisanby SH. Electroconvulsive therapy stimulus parameters: rethinking dosage. J ECT. 2010;26:159-174.

14. Grimm S, Beck J, Schuepbach D, Hell D, Boesiger P, Bermpohl F, et al. Imbalance between left and right dorsolateral prefrontal cortex in major depression is linked to negative emotional judgment: an fMRI study in severe major depressive disorder. Biol Psychiatry. 2008;63:369-376.

15. Miller MD, Paradis CF, Houck PR, Mazumdar S, Stack JA, Rifai AH, et al. Rating chronic medical illness burden in geropsychiatric practice and research: application of the Cumulative Illness Rating Scale. Psychiatry Res. 1992;41:237-248.

16. Oquendo MA, Baca-Garcia E, Kartachov A, Khait V, Campbell CE, Richards M, et al. A computer algorithm for calculating the adequacy of antidepressant treatment in unipolar and bipolar depression. J Clin Psychiatry. 2003;64:825-833.

17. Oquendo MA, Malone KM, Ellis SP, Sackeim HA, Mann JJ. Inadequacy of antidepressant treatment for patients with major depression who are at risk for suicidal behavior. Am J Psychiatry. 1999;156:190-194.

18. Nasreddine ZS, Phillips NA, Bedirian V, Charbonneau S, Whitehead V, Collin I, et al. The montreal cognitive assessment, MoCA: a brief screening tool for mild cognitive impairment. J Am Geriatr Soc. 2005;53:695-699.

19. Wechsler D. Wechsler test of adult reading. San Antonio, TX: WTAR; 2001.

20. Patterson J. Controlled oral word association test. In: Kreutzer JS, DeLuca J. editors. Encyclopedia of clinical neuropsychology. New York, NY: Springer; 2001.

21. Nuechterlein KH, Green MF, Kern RS, Baade LE, Barch DM, Cohen JD, et al. The MATRICS consensus cognitive battery, part 1: test selection, reliability, and validity. Am J Psychiatry. 2008;165:203-213.

22. Trenerry MR, Crosson B, DeBoe J, Leber WR. Stroop neuropsychological screening test. Odessa, FL: Psychological Assessment Resources; 1989.
23. McElhiney MC, Moody BJ, Sackeim HA. The autobiographical memory interview - short form. In: Psychiatry DoB, editor. New York: New York State Psychiatric Institute; 2001.

24. Kayser S, Bewernick BH, Grubert C, Hadrysiewicz BL, Axmacher N, Schlaepfer TE. Antidepressant effects, of magnetic seizure therapy and electroconvulsive therapy, in treatment-resistant depression. J Psychiatr Res. 2011:45:569-576.

25. Sackeim HA, Prudic J, Nobler MS, Fitzsimons L, Lisanby SH, Payne N, et al. Effects of pulse width and electrode placement on the efficacy and cognitive effects of electroconvulsive therapy. Brain Stimul. 2008;1:71-83.

26. Backhouse FA, Noda Y, Knyahnytska Y, Farzan F, Downar J, Rajji TK, et al. Characteristics of ictal EEG in magnetic seizure therapy at various stimulation frequencies. Clin Neurophysiol. 2018;129:1770-1779.

27. Berlim MT, van den Eynde F, Tovar-Perdomo S, Daskalakis ZJ. Response, remission and drop-out rates following high-frequency repetitive transcranial magnetic stimulation (rTMS) for treating major depression: a systematic review and metaanalysis of randomized, double-blind and sham-controlled trials. Psychol Med. 2014;44:225-239.

28. Kellner CH, Husain MM, Knapp RG, McCall WV, Petrides G, Rudorfer MV, et al. Right unilateral ultrabrief pulse ECT in geriatric depression: phase 1 of the PRIDE study. Am J Psychiatry. 2016;173:1101-1109.

29. Semkovska M, McLoughlin DM. Objective cognitive performance associated with electroconvulsive therapy for depression: a systematic review and meta-analysis. Biol Psychiatry. 2010;68:568-577.

30. Kessler U, Schoeyen HK, Andreassen OA, Eide GE, Malt UF, Oedegaard KJ, et al. The effect of electroconvulsive therapy on neurocognitive function in treatmentresistant bipolar disorder depression. J Clin Psychiatry. 2014;75:e1306-1313.

31. Polster JD, Kayser S, Bewernick BH, Hurlemann R, Schlaepfer TE. Effects of electroconvulsive therapy and magnetic seizure therapy on acute memory retrieval. J ECT. 2015;31:13-19. 